\title{
Roles of FGF20 in dopaminergic neurons and Parkinson's disease
}

\author{
Nobuyuki Itoh * and Hiroya Ohta \\ Department of Genetic Biochemistry, Kyoto University Graduate School of Pharmaceutical Sciences, Kyoto, Japan
}

\section{Edited by:}

Volker Eulenburg, University of

Erlangen 1054, Germany

\section{Reviewed by:}

Marcos R. Costa, Federal University

of Rio Grande do Norte, Brazil

Daniele Bottai, University of Milan,

Italy

Patrick Schloss, Central Institute of

Mental Health, Germany

\section{*Correspondence:}

Nobuyuki Itoh, Department of

Genetic Biochemistry, Kyoto

University Graduate School of

Pharmaceutical Sciences,

Yoshida-shimoadachi, Sakyo,

Kyoto 606-8501, Japan

e-mail: itohnobu@

pharm.kyoto-u.ac.jp
The fibroblast growth factor (FGF) family comprises 22 members with diverse functions in development and metabolism. Fgf20 was originally identified as a new Fgf preferentially expressed in the substantia nigra pars compacta (SNpc). Fgf20, which acts on proximal cells, significantly enhanced the survival of cultured dopaminergic neurons by activating the mitogen-activated protein kinase (MAPK) pathway through Fgf receptor 1c. In the rat model of Parkinson's disease, Fgf20 afforded significant protection against the loss of dopaminergic neurons. The significant correlation of Parkinson's disease with single-nucleotide polymorphisms in FGF20 indicates that the genetic variability of FGF20 can be a Parkinson's disease risk. Neural and embryonic stem (ES) cells have been considered as cell resources for restorative transplantation strategies in Parkinson's disease. Fgf20 promoted the differentiation of these stem cells into dopaminergic neurons, which attenuated neurological symptoms in animal models of Parkinson's disease. These findings indicate the importance of FGF20 for the differentiation and survival of dopaminergic neurons and the etiology and therapy of Parkinson's disease.

Keywords: dopaminergic neurons, Fgf, Fgf20, Parkinson's disease, stem cells, SNP

\section{INTRODUCTION}

Fibroblast growth factors (FGFs) are polypeptides with diverse functions in development, metabolism, and neural activities. The FGF family comprises 22 members, which have been classified as paracrine, endocrine, and intracrine FGFs by their mechanisms of action. Most FGFs are paracrine FGFs that act as local signaling molecules (Itoh and Ornitz, 2011). Fgf20 was originally identified as a new Fgf preferentially expressed in the substantia nigra pars compacta (SNpc). Fgf20, a paracrine Fgf, with neurotrophic activity in cultured dopaminergic neurons (Ohmachi et al., 2000, 2003) has been suggested to play important roles in the development of dopaminergic neurons (Grothe et al., 2004; Takagi et al., 2005; Correia et al., 2007; Shimada et al., 2009). In addition, FGF20 mutations may result in Parkinson's disease (van der Walt et al., 2004; Satake et al., 2007; IPDGC, 2011; Pan et al., 2012; Pihlstrøm et al., 2013; Wang et al., 2013). As these findings indicate that FGF20 may provide useful clues on the etiology and therapy of Parkinson's disease, a succinct review on the roles of FGF20 in dopaminergic neurons and Parkinson's disease has been provided. In this review, we refer to the human and rodent orthologs as FGF20 and Fgf20 according to the Human Genome Organization and the Mouse Genome Informatics, respectively.

\section{IDENTIFICATION OF FGF20}

Fgf20, originally identified in the rat brain, encodes a secreted protein of 212 amino acids (Ohmachi et al., 2000). The FGF gene family comprising 22 members has been classified into 7 subfamilies; FGF/1/2, FGF4/5/6, FGF3/7/10/22, FGF8/17/18, FGF9/16/20, FGF11/12/13/14, and FGF19/21/23. FGF20 is a member of the FGF9/16/20 subfamily, which is a paracrine Fgf (Figure 1) (Itoh and Ornitz, 2011).

\section{FGF2O IN DOPAMINERGIC NEURON SURVIVAL}

As paracrine FGFs are local signal molecules, Fgf20 is expected to act on dopaminergic neurons in the $\mathrm{SNpc}$ in a paracrine manner. However, as both Fgf20 and Fgfr1 are expressed in most dopaminergic neurons in the SNpc, Fgf20 may act on them in an autocrine/paracrine manner. Fgf20 was shown to significantly enhance the survival of cultured rat dopaminergic neurons (Ohmachi et al., 2000, 2003).

Paracrine FGF signaling is mediated by the activation of FGFR. Paracrine FGFs bind to FGFRs and induce the phosphorylation of specific cytoplasmic tyrosine residues, which triggers the activation of cytoplasmic signal transduction pathways. Major FGF/FGFR-dependent signaling was shown to be mediated by the mitogen-activated protein kinase (MAPK) and phospholipase- $\mathrm{C} \gamma$ pathways (Thisse and Thisse, 2005).

FGFRs are receptor tyrosine kinases with an extracellular ligand-binding domain, which comprises three immunoglobulinlike domains (I, II, and III). There are seven major FGFR proteins including FGFRs1b, 1c, 2b, 2c, 3b, 3c, and 4, which are generated from four functional FGFR genes, FGFR1-FGFR4 by alternative splicing (Zhang et al., 2006). Fgfrlc is abundantly expressed in dopaminergic neurons in the SNpc. Fgf20 binds to Fgfr1c with high affinity. Experiments with the Fgfr inhibitor SU5402 or MAPK pathway inhibitor PD98059 indicate that activation of the MAPK pathway by Fgf20 through Fgfr1c is essential for the survival of dopaminergic neurons in the SNpc (Figure 2) (Ohmachi et al., 2003).

Calbindin-negative dopaminergic neurons are preferentially lost in Parkinson's disease. Fgf20 almost completely rescued rat calbindin-negative midbrain dopaminergic neurons from the toxicity of 6-hydroxyldopamine and stress-induced cytosolic 

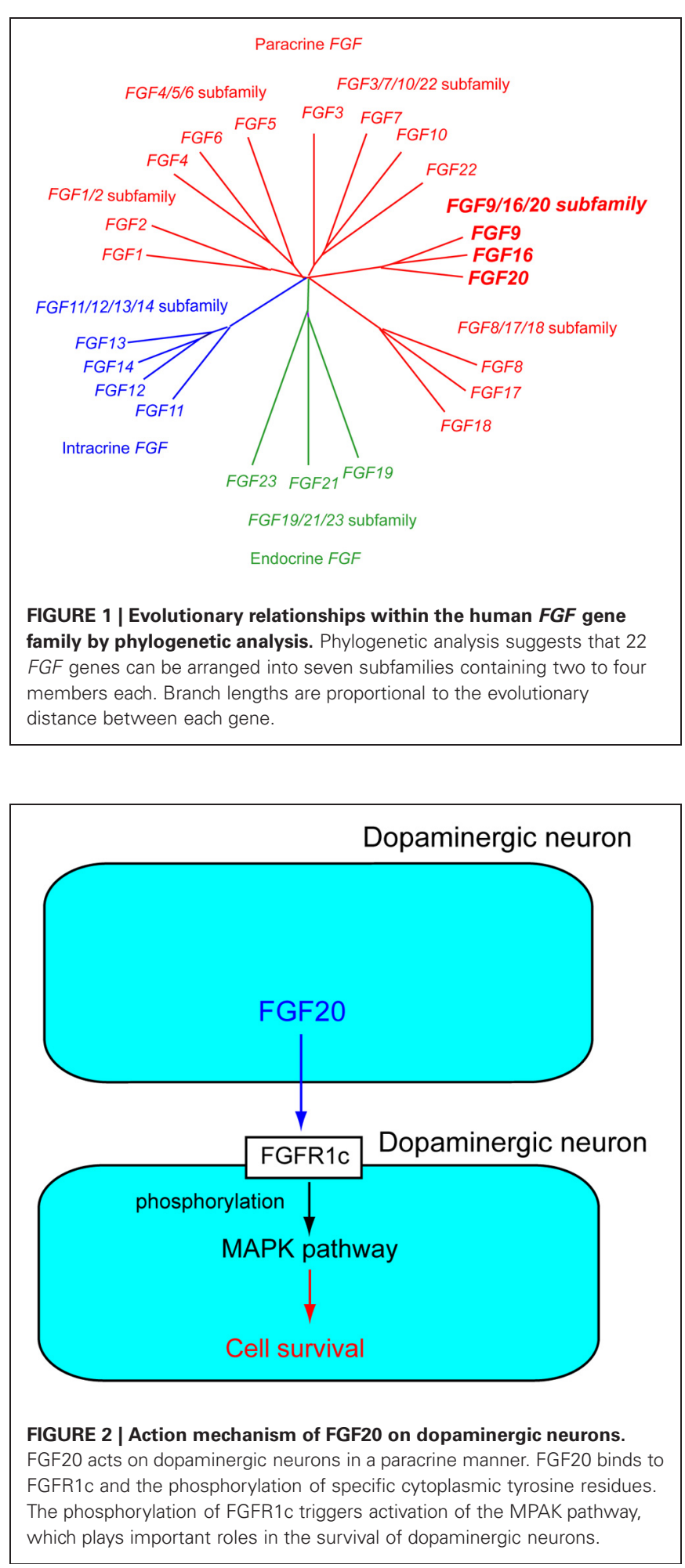

dopamine, and promoted dopamine release in calbindin-negative dopaminergic neurons by activating Fgfr 1 followed by its downstream cascade activation. These results show that Fgf20 protects the specific midbrain neuron type at most risk in Parkinson's patients (Murase and McKay, 2006).
In the unilateral, 6-hydroxydopamine lesion rat model of Parkinson's disease, supranigral infusion of Fgf20 afforded significant protection against the loss of dopaminergic neurons in the SNpc and striatum. Protection of the nigrostriatal tract was accompanied by the significant preservation of gross locomotion and fine motor movements and the reversal of apomorphineinduced contraversive rotations. These results imply the potential neuroprotective role of Fgf20 in this disease (Sleeman et al., 2012).

\section{FGF2O IN PARKINSON'S DISEASE}

Parkinson's disease is a common neurodegenerative disorder. The inability to control movement in patients with this disease has been attributed to the severe loss of dopaminergic neurons within the substantia nigra. Environmental and genetic sources act together in the disease cascade.

FGF20 has been mapped to $8 \mathrm{p} 21.3-8 \mathrm{p} 22$, which is within an area of Parkinson's disease linkage. To test whether FGF20 genetic variability was a risk factor for Parkinson's disease, five single-nucleotide polymorphisms (SNPs) lying in FGF20 were examined in a large family study. The highly significant correlation of Parkinson's disease with one SNP located in the intron and two SNPs in the $3^{\prime}$ regulatory region was revealed, which indicated that $F g f 20$ genetic variability is a risk factor for Parkinson's disease (van der Walt et al., 2004). In addition, FGF20 genetic variability was shown to be a risk factor for Parkinson's disease in Japanese and Chinese populations (Satake et al., 2007; Pan et al., 2012), while, was not a risk factor for Parkinson's disease in Finnish and Greek populations (Clarimon et al., 2005). The discrepancy between these results remains to be elucidated.

The SNP in the $3^{\prime}$ non-coding region of FGF20 can be a risk factor for Parkinson's disease. The risk allele disrupts a binding site for microRNA-433, increasing FGF20 mRNA translation. This increase in FGF20 mRNA translation has been correlated with increased $\alpha$-synuclein expression. As $\alpha$-synuclein is the principal component of filamentous Lewy bodies, the defining pathological hallmark of Parkinson's disease, these findings suggest a novel mechanism of action for the risk of Parkinson's disease (Wang et al., 2008). In addition, FGF20 and $\alpha$-synuclein were also shown to be associated with sporadic Parkinson's disease (Mizuta et al., 2008). However, no association between the SNP in FGF20, microRNA-433, or $\alpha$-synuclein expression and Parkinson's disease have been reported (Wider et al., 2009; de Mena et al., 2010). The discrepancy between these results remains to be elucidated.

The genetic variability of the monoamine oxidase $\mathrm{B}$ gene $(M A O B)$ has also been suggested as a risk factor for Parkinson's disease. Both FGF20 and MAOB are in the dopamine biopathway. SNP variants in FGF20 and MAOB show evidence of statistical interactions, which emphasizes the importance of considering them jointly in the genetic analysis of Parkinson's disease, and illustrates the potential patterns of biological interactions contributing to the risk of Parkinson's disease (Gao et al., 2008).

A genome-wide association study (GWAS) to examine many common genetic variants was conducted in different individuals to identify any variant associated with a trait. The GWAS typically focused on associations between SNPs and traits such as major 
diseases. FGF20 was shown to be a risk factor for Parkinson's disease by the GWAS (IPDGC, 2011; Pihlstrøm et al., 2013; Wang et al., 2013).

\section{FGF2O IN THE NEURAL DIFFERENTIATION OF STEM CELLS INTO DOPAMINERGIC NEURONS}

Neural stem (NS) cells are multipotent cells characterized by their capability to differentiate into neurons, astrocytes, and oligodendrocytes, and have been considered as cell resources for restorative transplantation strategies in Parkinson's disease. Nurr1 is a transcription factor of the thyroid hormone/retinoic acid nuclear receptor superfamily that is required for the induction of dopaminergic neurons. However, Nurr1 alone is not sufficient to induce a dopaminergic phenotype in NS cells. A co-culture of Nurr1-transfected NS cells with Schwann cells overexpressing $\mathrm{Fgf2O}$ was shown to induce dopaminergic neurons in NS cells. Differentiated Nurr1-NS cells retained both neuronal morphology and tyrosine hydroxylase expression after transplantation into the striatum of 6-hydroxydopamine-lesioned rats. However, neuritogenesis was only observed after postnatal grafts. These results suggest that Fgf20 promotes the differentiation of Nurr1-NS cells into dopaminergic neurons and that additional factors are required for the efficient differentiation of dopaminergic neurons in the adult brain (Grothe et al., 2004).

Embryonic stem (ES) cells are pluripotent cells derived from the inner cell mass of the preimplantation blastocyst. These cells have many of the characteristics required of a cell source for cell-replacement therapy, including proliferation and differentiation capacities. ES cells are also promising donor cell sources for cell-replacement therapy in Parkinson's disease. FGF20 acts synergistically with FGF2 to increase the number of dopaminergic neurons in primate ES cell-derived neurospheres composed of neural progenitors. Dopaminergic neurons generated from primate ES cells were transplanted into 1-methyl-4-phenyl-1,2,3,6tetrahydropyridine-treated (MPTP-treated) primates, a primate model for Parkinson's disease. Behavioral studies and functional imaging revealed that the transplanted dopaminergic cells functioned as dopaminergic neurons and attenuated MPTP-induced neurological symptoms (Takagi et al., 2005).

Parthenogenesis has attracted attention as an alternative method to derive ES cells that does not involve the destruction of viable embryos. Transplantation of dopaminergic neurons generated from parthenogenetic primate ES cells restored motor function in hemi-Parkinsonian, 6-hydroxy-dopamine-lesioned rats. Exposure to FGF20, along with WNT5a and FGF2, at the final

\section{REFERENCES}

Clarimon, J., Xiromerisiou, G., Eerola, J., Gourbali, V., Hellström, O., Dardiotis, E., et al. (2005). Lack of evidence for a genetic association between FGF20 and Parkinson's disease in Finnish and Greek patients. BMC Neurol. 5:11. doi: 10.1186/14712377-5-11

Correia, A. S., Anisimov, S. V., Roybon, L., Li, J. Y., and Brundin, P. (2007). Fibroblast growth factor-20 increases the yield of midbrain dopaminergic neurons derived from human embryonic stem cells. Front. Neuroanat. 1:4. doi: 10.3389/neuro.05.004.2007

de Mena, L., Cardo, L. F., Coto, E., Miar, A., Díaz, M., Corao, A. I., et al. (2010). FGF20 rs12720208 SNP and microRNA-433 variation: no association with Parkinson's disease in Spanish patients. Neurosci. Lett. 479, 22-25. doi: 10.1016/j.neulet. 2010.05.019

stage of in vitro differentiation enhanced the maturation and in vivo survival of dopaminergic neurons and, correspondingly, the extent of motor recovery in transplanted animals (SanchezPernaute et al., 2008). Induced pluripotent stem (iPS) cell-derived dopaminergic neurons were also shown to integrate into the striatum of Parkinsonian rats with behavioral improvements (Gibson et al., 2012). However, experiments on iPS cells using FGF20 have not been reported.

Neuronal differentiation in human ES cells was induced by co-culturing with PA6 mouse stromal cells. The number of tyrosine hydroxylase-expressing neurons significantly increased in culture medium supplemented with FGF20. Cultured cells also expressed other midbrain dopaminergic markers, which suggests that some differentiate into midbrain dopaminergic neurons. However, FGF20 has no effect on the size of the soma area or neurite length of dopaminergic neurons. FGF20 significantly reduced the proportion of cells undergoing cell death. These results indicate that FGF20 specifically increased the yield of dopaminergic neurons from human ES cells grown on PA6 feeder cells, and at least part of this effect was due to a reduction in cell death (Correia et al., 2007). In addition, FGF20 along with FGF2 enhanced dopaminergic neuron differentiation from human ES cell-derived neural progenitor cells directly without co-culturing with PA6 cells (Shimada et al., 2009).

\section{CONCLUSIONS}

Fgf20 is expressed in the SNpc of the midbrain. Fgf20 significantly enhances the survival of cultured dopaminergic neurons in a paracrine manner. In the rat model of Parkinson's disease, Fgf20 affords significant protection against the loss of dopaminergic neurons. The significant correlation of Parkinson's disease with SNPs within FGF20 indicated that FGF20 genetic variability is a risk factor for Parkinson's disease. Fgf20 promotes differentiation of cultured cells into dopaminergic neurons, and attenuated neurological symptoms in animal models of Parkinson's disease. These findings indicate the importance of FGF20 in both the differentiation and survival of dopaminergic neurons and the etiology and therapy of Parkinson's disease. Further studies on FGF20 will provide useful clues on the etiology and therapy of Parkinson's disease.

\section{ACKNOWLEDGMENTS}

This work was supported by a Grant-in-aid for Scientific Research from the Ministry of Education, Science, Culture and Sports of Japan.

Gao, X., Scott, W. K., Wang, G., Mayhew, G., Li, Y. J., Vance, J. M., et al. (2008). Gene-gene interaction between FGF20 and MAOB in Parkinson disease. Ann. Hum. Genet. 72, 157-162. doi: 10.1111/j.1469-1809.2007.00418.x

Gibson, S. A., Gao, G. D., McDonagh, K., and Shen, S. (2012). Progress on stem cell research towards the treatment of Parkinson's disease. Stem Cell Res. Ther. 3, 11. doi: $10.1186 /$ scrt102
Grothe, C., Timmer, M., Scholz, T., Winkler, C., Nikkhah, G., Claus, P., et al. (2004). Fibroblast growth factor-20 promotes the differentiation of Nurr1-overexpressing neural stem cells into tyrosine hydroxylase-positive neurons. Neurobiol. Dis. 17, 163-170. doi: 10.1016/j.nbd.2004.07.007

International Parkinson's Disease Genomics Consortium (IPDGC); Wellcome Trust Case Control Consortium 2 (WTCCC2). (2011). 
A two-stage meta-analysis identifies several new loci for Parkinson's disease. PLoS Genet. 7:e1002142. doi: 10.1371/journal.pgen.1002142

Itoh, N., and Ornitz, D. M. (2011). Fibroblast growth factors: from molecular evolution to roles in development, metabolism and disease. J. Biochem. 149, 121-130. doi: $10.1093 / \mathrm{jb} / \mathrm{mvq} 121$

Mizuta, I., Tsunoda, T., Satake, W., Nakabayashi, Y., Watanabe, M., Takeda, A., et al. (2008). Calbindin 1, fibroblast growth factor 20, and alpha-synuclein in sporadic Parkinson's disease. Hum. Genet. 124, 89-94. doi: 10.1007/s00439-008-0525-5

Murase, S., and McKay, R. D. (2006). A specific survival response in dopamine neurons at most risk in Parkinson's disease. J. Neurosci. 26, 9750-9760. doi: 10.1523/JNEUROSCI.2745-06.2006

Ohmachi, S., Mikami, T., Konishi, M., Miyake, A., and Itoh, N. (2003). Preferential neurotrophic activity of fibroblast growth factor-20 for dopaminergic neurons through fibroblast growth factor receptor1c. J. Neurosci. Res. 72, 436-443. doi: 10.1002/jnr.10592

Ohmachi, S., Watanabe, Y., Mikami, T., Kusu, N., Ibi, T., Akaike, A., et al. (2000). FGF-20, a novel neurotrophic factor, preferentially expressed in the substantia nigra pars compacta of rat brain. Biochem. Biophys. Res. Commun. 277, 355-360. doi: 10.1006/bbrc. 2000.3675

Pan, J., Li, H., Wang, Y., Ma, J. F., Zhang, J., Wang, G., et al.
(2012). Fibroblast growth factor 20 (FGF20) polymorphism is a risk factor for Parkinson's disease in Chinese population. Parkinsonism Relat. Disord. 18, 629-631. doi: 10.1016/j.parkreldis.2012.01.017

Pihlstrøm, L., Axelsson, G., Bjørnarå, K. A., Dizdar, N., Fardell, C., Forsgren, L., et al. (2013). Supportive evidence for 11 loci from genome-wide association studies in Parkinson's disease. Neurobiol. Aging 34, 1708.e7-1708.e13. doi: 10.1016/j.neurobiolaging.2012. 10.019

Sanchez-Pernaute, R., Lee, H., Patterson, M., Reske-Nielsen, C., Yoshizaki, T., Sonntag, K. C., et al. (2008). Parthenogenetic dopamine neurons from primate embryonic stem cells restore function in experimental Parkinson's disease. Brain 131, 2127-2139. doi: 10.1093/brain/awn144

Satake, W., Mizuta, I., Suzuki, S., Nakabayashi, Y., Ito, C., Watanabe, M., et al. (2007). Fibroblast growth factor 20 gene and Parkinson's disease in the Japanese population. Neuroreport 18, 937-940. doi: 10.1097/WNR.0b013e328133265b

Shimada, H., Yoshimura, N., Tsuji, A., and Kunisada, T. (2009). Differentiation of dopaminergic neurons from human embryonic stem cells: modulation of differentiation by FGF-20. J. Biosci. Bioeng. 107, 447-454. doi: 10.1016/j.jbiosc.2008.12.013

Sleeman, I. J., Boshoff, E. L., and Duty, S. (2012). Fibroblast growth factor-20 protects against dopamine neuron loss in vitro and provides functional protection in the 6-hydroxydopaminelesioned rat model of Parkinson's disease. Neuropharmacology 63, 1268-1277. doi: 10.1016/j.neuropharm.2012.07.029

Takagi, Y., Takahashi, J., Saiki, H., Morizane, A., Hayashi, T., Kishi, Y., et al. (2005). Dopaminergic neurons generated from monkey embryonic stem cells function in a Parkinson primate model. J. Clin. Invest. 115, 102-109. doi: 10.1172/JCI21137

Thisse, B., and Thisse, C. (2005). Functions and regulations of fibroblast growth factor signaling during embryonic development. Dev. Biol. 287, 390-402. doi: 10.1016/j.ydbio.2005.09.011

van der Walt, J. M., Noureddine, M. A. Kittappa, R., Hauser, M. A., Scott, W. K., McKay, R., et al. (2004). Fibroblast growth factor 20 polymorphisms and haplotypes strongly influence risk of Parkinson disease. Am. J. Hum. Genet. 74, 1121-1127. doi: $10.1086 / 421052$

Wang, G., van der Walt, J. M., Mayhew, G., Li, Y. J., Züchner, S., Scott, W. K., et al. (2008). Variation in the miRNA-433 binding site of FGF20 confers risk for Parkinson disease by overexpression of alpha-synuclein. Am. J. Hum. Genet. 82, 283-289. doi: 10.1016/j.ajhg.2007.09.021

Wang, M., Wang, L., Jiang, N., Jia, T., and Luo, Z. (2013). A robust and efficient statistical method for genetic association studies using case and control samples from multiple cohorts. BMC Genomics 14:88. doi: 10.1186/1471-2164-14-88
Wider, C., Dachsel, J. C., Soto, A. I., Heckman, M. G., Diehl, N. N., Yue, M., et al. (2009). FGF20 and Parkinson's disease: no evidence of association or pathogenicity via alpha-synuclein expression. Mov. Disord. 24, 455-459. doi: $10.1002 / \mathrm{mds} .22442$

Zhang, X., Ibrahimi, O. A., Olsen, S. K., Umemori, H., Mohammadi, M., and Ornitz, D. M. (2006). Receptor specificity of the fibroblast growth factor family. The complete mammalian FGF family. J. Biol. Chem. 281, 15694-15700. doi: 10.1074/jbc.M601252200

Conflict of Interest Statement: The authors declare that the research was conducted in the absence of any commercial or financial relationships that could be construed as a potential conflict of interest.

Received: 16 April 2013; paper pending published: 27 April 2013; accepted: 16 May 2013; published online: 31 May 2013.

Citation: Itoh $N$ and Ohta $H$ (2013) Roles of FGF2O in dopaminergic neurons and Parkinson's disease. Front. Mol. Neurosci. 6:15. doi: 10.3389/fnmol. 2013.00015

Copyright (c) 2013 Itoh and Ohta. This is an open-access article distributed under the terms of the Creative Commons Attribution License, which permits use, distribution and reproduction in other forums, provided the original authors and source are credited and subject to any copyright notices concerning any third-party graphics etc. 